\title{
Application of Tatian's Method to Slanted-Edge MTF Measurement
}

\author{
Peter D. Burns* \\ Eastman Kodak Company, Rochester, NY USA 14650-1925
}

\begin{abstract}
The ISO 12233 method for the measurement of the spatial frequency response (SFR) of digital still cameras and scanners is based on the analysis of slanted-edge image features. The procedure applies a form of edge-gradient analysis to an estimated edge-spread function. As with all measurement, image noise can introduce bias error and variation into the resulting camera SFR and modulation transfer function, (MTF). It is often pointed out that applying a derivative filter to the estimated edge-spread function, as is done in the ISO method, amplifies this image noise. To reduce the influence of noise on the measurement, data averaging and fitting have been proposed. One method for edge-gradient analysis, reported by Tatian, avoids the above discrete derivative step. The MTF is expressed as a trigonometric series, whose elements are estimated from the measured edge-spread function.

We describe the application of this method as an intermediate step in the ISO procedure. The method was benchmarked for both synthetic edges and captured test images. Results indicate good agreement between the two estimates for lownoise image data. For higher noise levels, however, Tatian's method is found to be susceptible to the selection (cropping) of the input data array. For the conditions tested, we found no clear advantage of this method over the current ISO procedure. For other applications, the slanted-edge analysis provides a front end to Tatian's method, and others, based on parametric modeling or statistical fitting.
\end{abstract}

Keywords: SFR, presampling MTF, edge-spread function, image quality

\section{INTRODUCTION}

For image capture systems, the modulation transfer function (MTF) provides a description of the efficiency of image detail acquisition from object to recorded image. ${ }^{1}$ This frequency-domain measure is well suited to overall system evaluation and analysis of subsystem design. MTF measurement usually relies on the analysis of continuous or sampled images of various test targets or objects. Methods using periodic signals, random noise fields, narrow slit exposures, and edge features are well established. When simple target generation and a limited spatial extent are desirable, edgegradient analysis methods area often used. ${ }^{2,3}$ In this paper, we describe how a particular method, described by Tatian, ${ }^{4}$ might be applied within the context of a current standard measurement procedure used for digital camera and scanner evaluation.

\subsection{ISO 12233 Method}

The ISO method, ${ }^{5}$ based on analysis of slanted-edge image features, is a special case of edge-gradient analysis. ${ }^{6-8}$ The basic steps for edge-gradient methods are shown in Fig. 1, where the input is the system response to a high quality edge feature. For a digital camera or scanner, this would be the digital image of a printed test target of an edge or scene object. From an edge image, one needs to estimate an edge-spread function for the image transition. From this edge profile, a line-spread function is found by estimating the first derivative. The modulus of the Fourier transform of this function, after scaling, is the measured normalized signal modulation function of spatial frequency. In general, the output modulation is divided by the corresponding input edge (test target) modulation, frequency-by-frequency, to yield the measured system MTF. We will refer to the single output modulation, normalized to unity at zero frequency, as the spatial frequency response (SFR), consistent with the standard. The ratio will be called the estimated or measured MTF.

One particular variant of this method, based on a rotated- or slanted-edge feature, is called for the ISO 12233 standard for digital still camera resolution evaluation. The slanted-edge method has the advantage of being insensitive to the exact alignment of the edge feature to the image-sampling lattice. The edge profile is computed in three steps, as shown in the left-hand side of Fig. 2. First the edge location and direction are estimated from this image array data via a linear

Proc. SPIE-IS\&T Electronic Imaging Symposium, SPIE vol. 5668, 255-261, 2005.

* now with Burns Digital Imaging 
equation. This is found after taking a one-dimensional discrete derivative and finding the centroid for each data line. The image data for all pixels are projected along the direction of the edge to form a one-dimensional "super sampled" edge-spread function. A four-times over-sampling accomplished by this step reduces the influence of image aliasing of the measured SFR. After application of a Hamming window, the discrete Fourier transform is computed. The normalized modulus of this transform array is taken as the $\mathrm{SFR}_{1}$

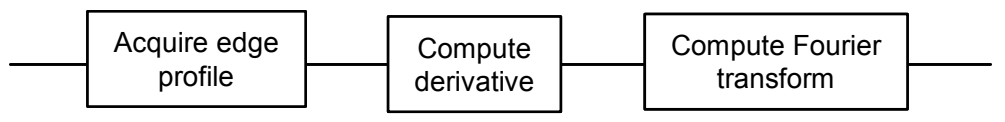

Figure 1: Steps in edge-gradient MTF analysis

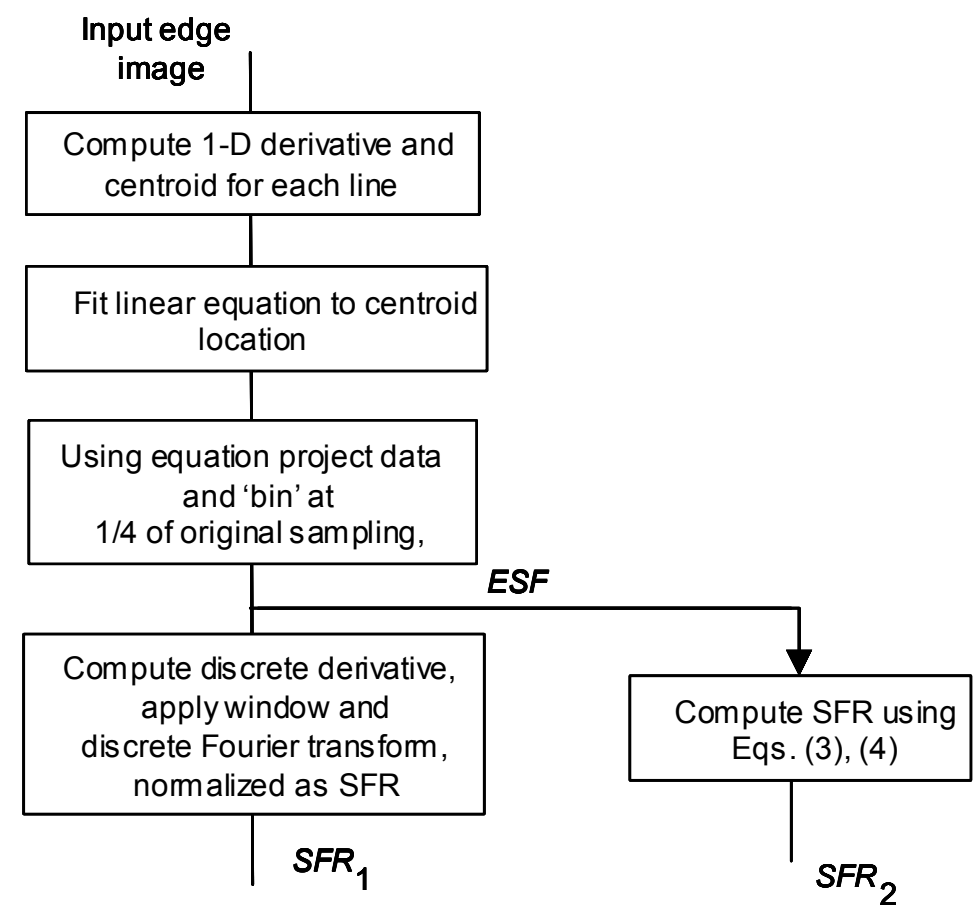

Figure 2: Applying Tatian's method to the ESF, found as an intermediate step in the ISO method

\subsection{Tatian's Method}

Tatian described a method that effectively combines the last two steps of Fig. 1 into a single operation. For sampled edge spread function (ESF) data, $e(x)$, he assumes that the signal is band-limited. The ESF is usually scaled so its range is $[0,1]$, and separated into its even and odd parts, $e_{1}(x)$ and $e_{2}(x)$. Since $e$ is a real function, its Fourier transform, $\mathrm{E}(f)$, can be expressed as

$$
\mathrm{E}(f)=\mathbf{F}\left\{e_{1}(x)+e_{1}(x)\right\}=\mathrm{E}_{1}(f)-i \mathrm{E}_{2}(f),
$$

where $\mathbf{F}$ indicates the Fourier transform and $f$ is spatial frequency. Our analysis will be for sampled signals,

$$
e(n \Delta x), e_{1}(n \Delta x), e_{2}(n \Delta x), \text { for } n=1,2, \ldots \mathrm{N},
$$

where $\Delta x$ and $\mathrm{N}$ are the sampling interval and number of data, respectively. Eq.(1) can be written as 


$$
\mathrm{E}(f)=\sum_{n=-\infty}^{\infty} e_{1}(n \Delta x) \cos (2 \pi f n \Delta x)-i \sum_{n=-\infty}^{\infty} e_{2}(n \Delta x) \sin (2 \pi f n \Delta x)
$$

The expression is evaluated for discrete frequencies of interest, $f_{1}, f_{2}, \ldots$, over the range $0 \leq f<(2 \Delta x)^{-1}$. Each summation term of Eq.(2) can be evaluated by considering the various regions where, $n<\mathrm{N}, \mathrm{N}<n<\mathrm{N}, n>\mathrm{N}$. Expressions are derived for the Fourier transform of the even and odd components of $e$. We are interested in the Fourier transform of the derivative of the ESF, however, and this is found using the property,

$$
\mathbf{F}\left\{\frac{\partial e(x)}{\partial x}\right\}=2 \pi i f \mathrm{E}(f) .
$$

The resulting expressions for the real and imaginary parts of the Fourier transform of the line-spread function are,

$$
\begin{gathered}
\Re\left[\mathbf{F}\left\{\frac{d e(x)}{d x}\right\}\right]=4 \pi f\left[\sum_{n=1}^{\mathrm{N}} e_{2}(n \Delta x) \sin (2 \pi f n \Delta x)\right]+\frac{\cos (2 \pi f \Delta x(\mathrm{~N}+0.5))}{\operatorname{sinc}(f \Delta x)} \\
\operatorname{Im}\left[\mathbf{F}\left\{\frac{d e(x)}{d x}\right\}\right]=4 \pi f\left[\sum_{n=1}^{\mathrm{N}} e_{1}(n \Delta x) \cos (2 \pi f n \Delta x)\right]-\frac{\sin (2 \pi f \Delta x(\mathrm{~N}+0.5))}{\operatorname{sinc}(f \Delta x)}+2 \pi f e_{1}(0) .
\end{gathered}
$$

The corresponding modulus and normalized SFR are given by,

$$
m(f)=\left(\mathfrak{R} \mathrm{e}\left[\mathbf{F}\left\{\frac{d e(x)}{d x}\right\}\right]^{2}+\operatorname{Im}\left[\mathbf{F}\left\{\frac{d e(x)}{d x}\right\}\right]^{2}\right)^{0.5}
$$

and

$$
\operatorname{SFR}_{2}(f)=m(f) / m(0)
$$

The above indicates how the SFR or MTF might be found from noise-free sampled edge spread function data. It does not, however, guarantee that this approach, when applied to actual digital image data, will result in a useful measurement. It is only when the accuracy and precision of the result are investigated that we can determine if, and under which conditions any particular method is to be recommended.

\section{NEW SLANT ON TATIAN}

Since the ESF is estimated as an intermediate step in the ISO method, this array was used as an input to the procedure based on Eqs, (3) and (4). This is shown on the right-hand side of Fig. 2. Note that for Tatian's method we have freedom to evaluate the SFR at any spatial frequency over the range $[0-0.5 / d]$, where $d$ is the sampling interval of the ESF array. Since the sampling of the ESF array is sampled at intervals one-fourth the size of the original image data, due to the binning step, the corresponding frequency range is $[0-2 / \Delta x]$, where the input image array was sampled at $\Delta x$. We will restrict our comparison to the more useful range of $[0-1 / \Delta x]$.

\subsection{Ideal Gaussian Edge}

For a noise-free Gaussian-shaped edge, both estimates were consistent, as shown in Fig. 3. For this direct comparison, Tatian's method was used to compute $\mathrm{SFR}_{2}$ at the same spatial frequencies for the ISO method,

$$
f_{n}=\frac{n}{\mathrm{~N} \Delta x}, n=0,1, \ldots, 2 \mathrm{~N},
$$


where $\mathrm{N}$ is the number of data for the original input test image file.

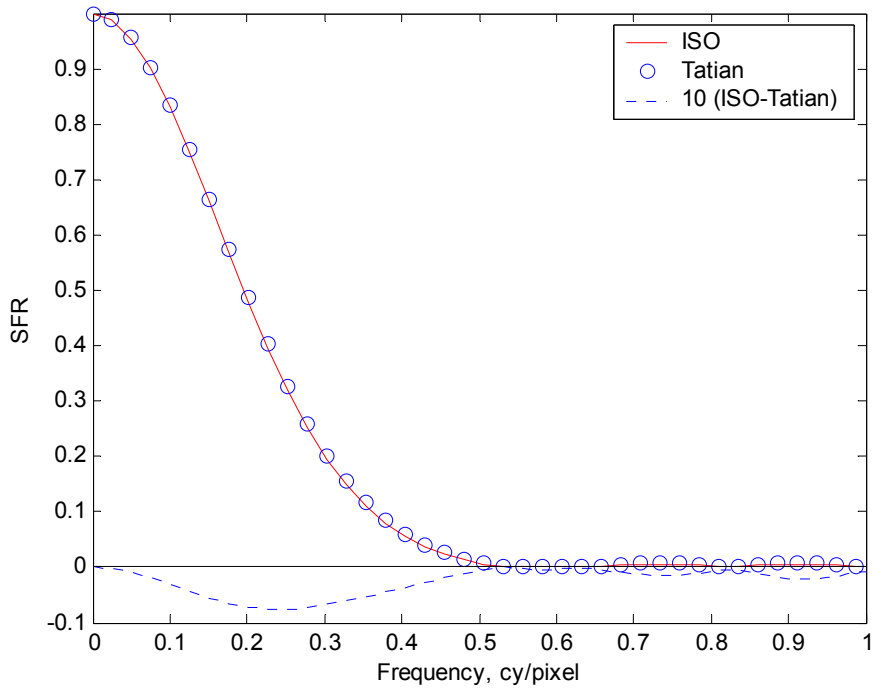

Figure 3: Results of ISO and Tatian methods for noise-free data, with 10X the difference also plotted

In a second comparison, random pixel fluctuations were added to the previous input image file. The noise added had a normal distribution, with $\sigma=3$, on a [0-255] signal range. These results, as shown in Fig. 4, indicate that the level of bias introduced by the noise at frequencies where there is low signal modulation, above $0.5 \mathrm{cy} / \mathrm{pixel}$, is about $2-3 \mathrm{X}$ that of $\mathrm{SFR}_{1}$.

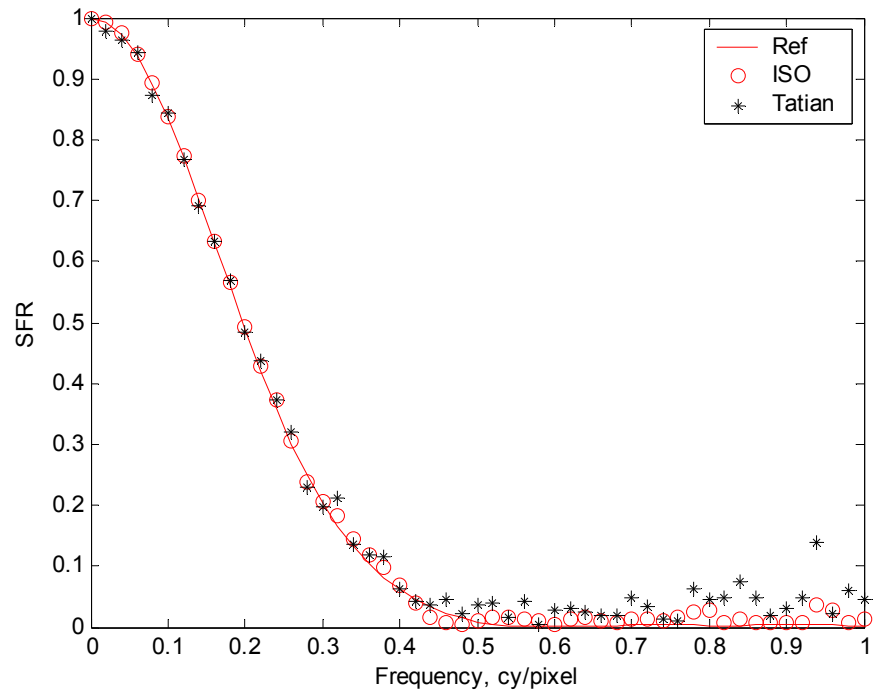

Figure 4: Results for the case shown in Fig. 3 with the addition of uncorrelated random noise (Ref = ISO noise-free), with $\mathrm{N}=50$.

The sensitivity of the measured SFR to the extent of the input data length was addressed next. This can happen when data far from the edge being analyzed are introducing error into the resulting SFR estimate. This arises in practical testing when, a region of interest (ROI) is chosen in a large digital image based on a computer monitor display. Tatian's method was applied to the test image ROI chosen in this way, with $\mathrm{N}=50$, and the number of lines along the edge was 
150. The method was then applied to the same edge image feature cropped to $\mathrm{N}=35$ and 25 input image pixels across the edge. The initial array was centered about the edge. Cropping the data did reduce the high-frequency bias error, and the shortest data length yielded results comparable to the ISO method. All SFR 2 estimates where evaluated at frequency intervals of $0.02 \mathrm{cy} / \mathrm{pixel}$. The results of this experiment are shown in Fig. 5. Tatian's method exhibited greater error than the ISO method, as also summarized in Table 1. The mean absolute and RMS error values are computed over all frequencies.

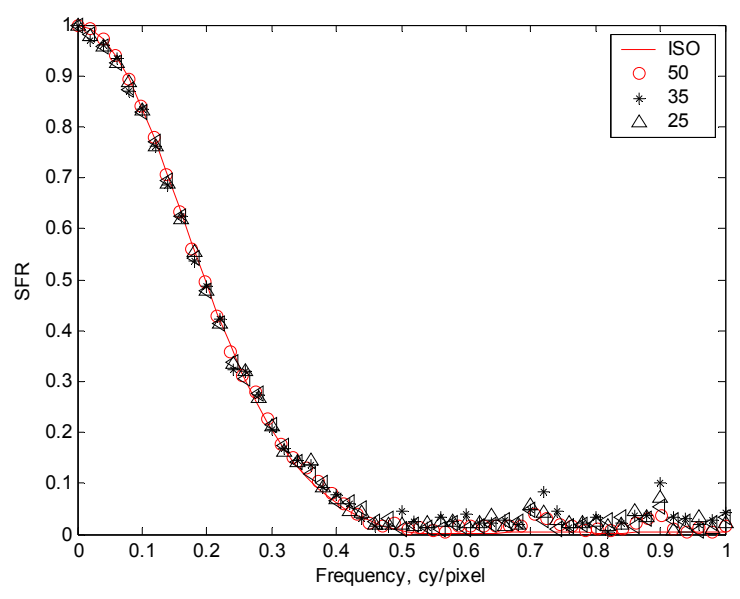

a.

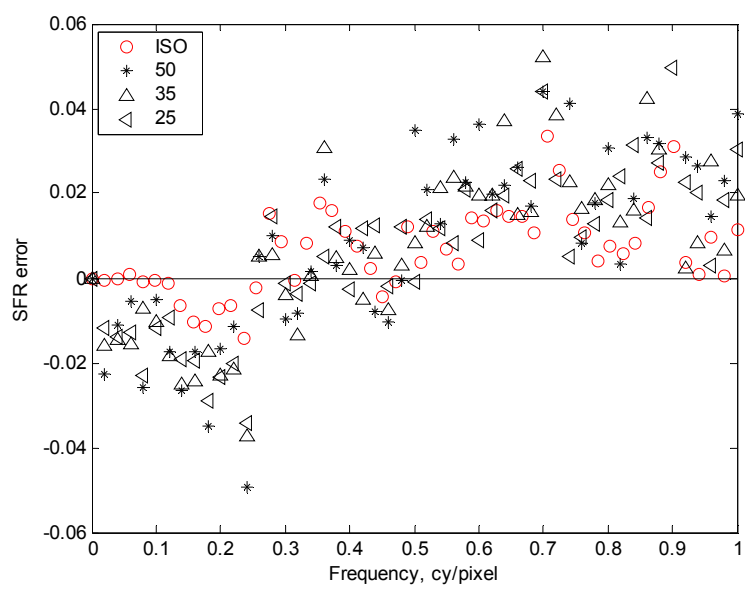

b.

Figure 5: a. SFR Results for the case shown in Fig. 4 with numbers of data, N. (Ref is noise-free).

b. shows differences from the noise-free result.

Table 1: Mean absolute, and RMS error statistics for the SFR estimates. $\mathrm{N}$ is the number of input image pixels across the edge.

\begin{tabular}{|c|c|c|c|c|}
\hline & ISO & \multicolumn{3}{|c|}{ Tatian } \\
\hline $\mathrm{N}$ & 50 & 50 & 35 & 25 \\
\hline Mean absolue & 0.0093 & 0.0222 & 0.0184 & 0.0161 \\
Rel. to ISO & 1.0000 & 2.3871 & 1.9785 & 1.7312 \\
\hline RMS & 0.0102 & 0.0264 & 0.0215 & 0.0183 \\
Rel. to ISO & 1.0000 & 2.5882 & 2.1078 & 1.7941 \\
\hline
\end{tabular}

\subsection{Digital Camera and Scanner Examples}

The previous results were for ideal edge images with the addition of uncorrelated stationary noise. Real digital image noise sources can deviate significantly from this case, and therefore may influence measurements results differently. A digital image from a desktop scanner was captured in a routine test for the standard SFR evaluation. The color scanner had a native sampling resolution off $600 \mathrm{dpi}$, and the test image was captured at $300 \mathrm{dpi}$, using a standard printed target. As before, the analysis data were chosen to include the edge feature and this array was used for both analysis methods. Results for the same data are shown in Fig. 6a. There is no noise-free ideal result available in this case; therefore, we simply observe that the ISO results appear to exhibit less variation from frequency-to-frequency. In addition, the highfrequency noise bias appears greater. Reducing the number of data for Tatian's method reduced the apparent frequencyto-frequency variation and bias; however, this may well be similar the spectral smoothing that we would expect from data truncation or other windowing. These results are shown in Fig. $6 b$.

The final comparison was for the evaluation of a consumer digital camera. The test image was captured in a studio with a large $(1 \mathrm{~m} \times 1 \mathrm{~m})$ gray printed target. The camera settings were those that are used by default. We would expect that the image noise statistics would differ from those of the scanner as a result of a different detector architecture and incamera image processing, such as color filter array interpolation and sharpening. The results are shown in Fig. 7, where 
the peak near $0.2 \mathrm{cy} /$ pixel suggests an image sharpening operation. Note the apparent underestimation of the SFR at low frequencies. In this case this error was not generally lower when the data length, N, was reduced.

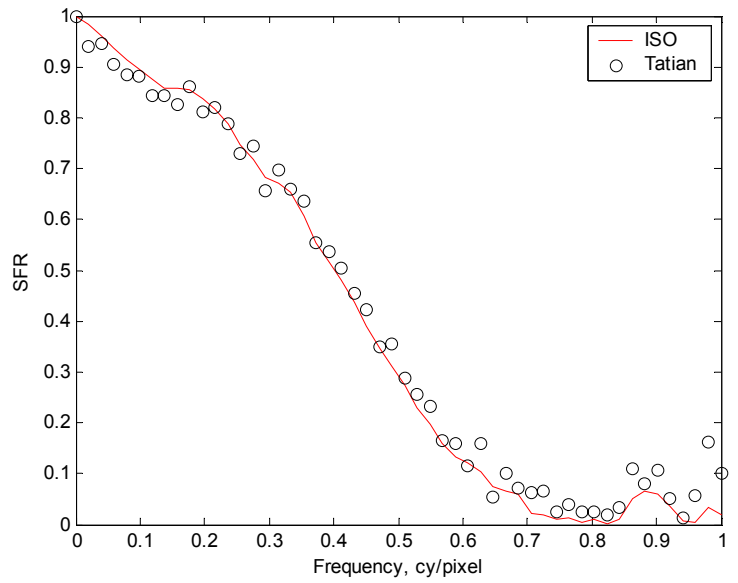

a.

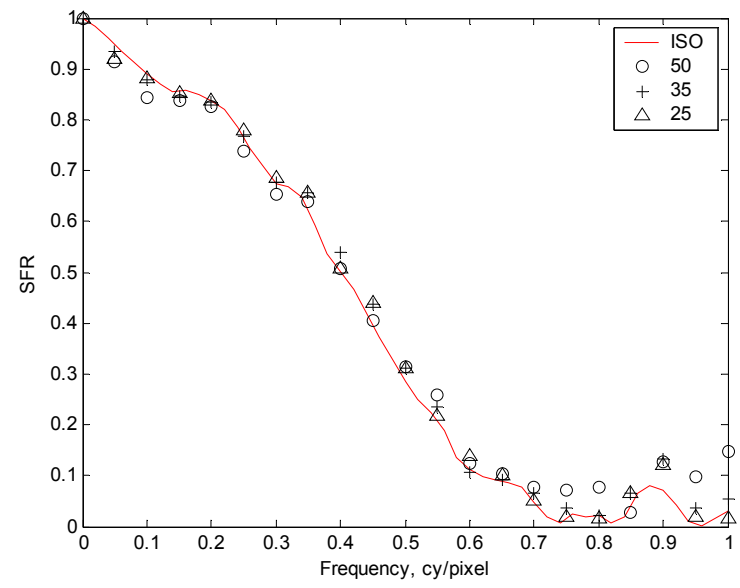

b.

Figure 6: Results for the digital scanner evaluation: a) direct comparison with each estimate computed at the same frequencies with large $\mathrm{N}=50$, and b) With $\mathrm{N}$ varying, evaluated at $\Delta f=0.05 \mathrm{cy} /$ pixel

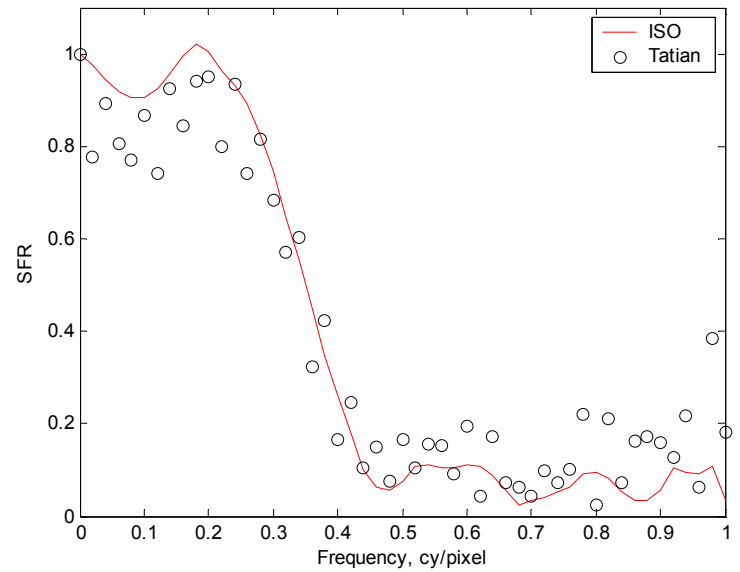

a.

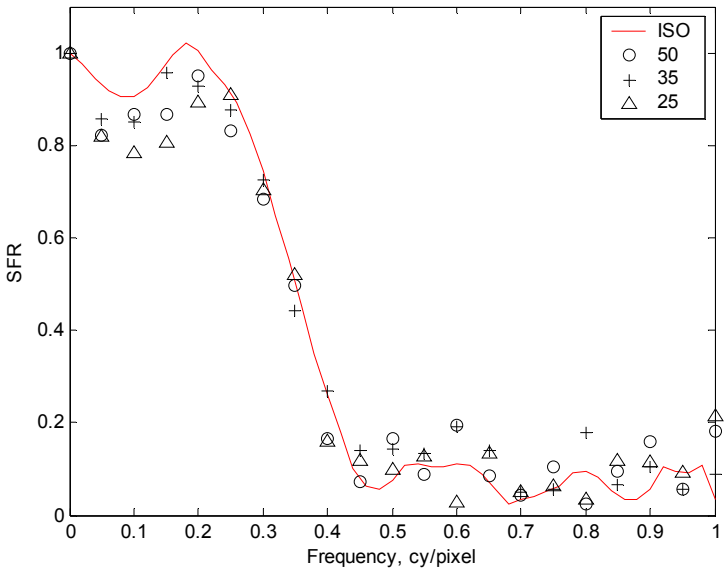

b.

Figure 7: Results for the digital camera evaluation: a) direct comparison with each estimate computed at the same frequencies with large $\mathrm{N}=50$, and b) With $\mathrm{N}$ varying, evaluated at $\Delta f=0.05 \mathrm{cy} /$ pixel

\section{SUMMARY}

Practical imaging system performance measurements are subject to errors of various sources, and MTF measurements are no exception. A reason often cited for avoiding the discrete derivative step in edge-gradient analysis is the noise amplification, evident in may applications of this method. The investigation of the use of Tatian's method in the context of the slanted-edge analysis indicated good agreement with the standard ISO method for low-noise data. Bias error and measurement fluctuations were observed, however, for data sets more characteristic of routine system measurements. Developing a measurement method into a tool for routine use involves the selection of various parameters and settings, and this optimization has not been attempted here. Nevertheless, for applications similar to those now common for the 
ISO method, Tatian's method does not appear to hold a clear advantage. It is likely that these the errors could be reduced by adaptive data selection, but these improvements might also carry over to other methods.

\section{REFERENCES}

1. C. J. Dainty and R. Shaw, Image Science, Ch. 5, Academic, New York, 1974.

2. F. Scott, R. M. Scott, and R. V. Shack, The Use of Edge Gradients in Determining Modulation-Transfer Function, Photogr. Sc. and Eng., 7, 64-68, 1963.

3. R. A. Jones, “An Automated Technique for Deriving MTFs from Edge Traces,” Photogr. Sc. and Eng., 11, 102-106, 1967.

4. B. Tatian, "Method for Obtaining the Transfer Function from the Edge Response Function," J. Opt. Soc. Amer., 55, 1014-1019, 1965.

5. ISO 12233, Photography - Electronic still picture cameras - Resolution measurements, ISO, 1998.

6. S. E. Reichenbach, S. K. Park, and R. Narayanswamy, “Characterizing Digital Image Acquisition Devices," Opt. Eng., 30, 170177, 1991.

7. E. Samei and M. J. Flynn, “A method for measuring the presampled MTF of digital radiographic systems using an edge Test device, Med. Phys., 25, 102-113, 1998.

8. P. D. Burns, "Slanted-Edge MTF for Digital Camera and Scanner Analysis," Proc. IS\&T PICS Conf., $135,2000$. 